Peppino come autore di favole e poesie è al centro dei saggi di Giuseppina Scognamiglio, la quale analizza i tratti distintivi della favola Pedrolino, "genere fra i più ardui ma in grado di fornire al suo autore il poetico pretesto per verificare una sua equilibrata filosofia di vita" (p. 100); di Loredana Palma, che illustra i vari contenuti delle favole in versi inserite nella raccolta Il fu Bobò (1954), e di Michele Sovente, il quale segue il filo che lega la produzione poetica di Peppino alla sua visione dell'uomo e del mondo che emerge dal suo stare sulla scena. Notevole è nelle opere di questo attore e autore la presenza della letteratura tout court, testimoniata - secondo Luigi Scorrano — dalle diverse reminiscenze che emergono nei dialoghi dei personaggi delle sue farse e commedie. Le abilità di Peppino come traduttore ed adattatore scaturiscono inoltre dal suo approccio al teatro di Plauto, che egli affronta mettendo in scena nel 1956 l'Aulularia e successivamente scrivendo l'atto unico Omaggio a Plauto, analizzati entrambi da Armando Rotondi.

Ad approfondire i legami che Peppino ha avuto con il cinema è Pasquale Iaccio, che ricostruisce il progressivo coinvolgimento cinematografico dei fratelli De Filippo a partire dagli anni Trenta, nonostante il giudizio critico del più giovane della famiglia nel confronti di quella che egli definiva come "un'arte morta". Sulla coppia comica di Totò e Peppino discute Ornella Petraroli, la quale mostra in che modo Massimo Troisi e Lello Arena abbiano vissuto un rapporto di complicità e fratellanza simile a quello che coinvolse i due grandi attori napoletani.

Il volume si conclude con le testimonianze di Manlio Santanelli, che riporta una confidenza fattagli da Peppino quando era assistente alla regia presso la RaiTv di Napoli e di Stefano De Stefano, secondo il quale sarebbe interessante vedere una rilettura del repertorio dell'autore fatta dai giovani teatranti contemporanei. Ed infine Annamaria Ackermann ricorda la sua esperienza nel ruolo di prima attrice al fianco di Peppino e Lucio Allocca rende nota una lettera manoscritta, inviatagli dal Maestro Commendatore nel 1977.

\title{
ANNALISA CASTELliTti
}

Università degli Studi di Napoli "Federico II"

\section{Ianuam linguarum reserare. Saggi in onore di Bona Cambiaghi. A cura di Cristina Bosisio. Milano: Mondadori ("Le Monnier Università"), 2011. Pp. 340. ISBN 978-88-00-74068-5. € 23.}

La pubblicazione di un Festschrift offre spesso l'occasione di apprezzare quanto l'attività scientifica e l'insegnamento di un docente abbiano influenzato, affascinato, stimolato allievi e colleghi, dando luogo, a loro volta, a nuove passioni e ricerche. E il caso del volume Ianuam linguarum reserare, che raccoglie trentanove contributi per onorare la carriera scientifica e accademica di Bona Cambiaghi; carriera più che quarantennale al momento della pubblicazione del volume collettaneo, dopo aver attraversato i territori della linguistica generale e francese, della linguistica applicata e della glottodidattica. Un percorso che ha avuto come punto di riferimento le università milanesi: dalla Bocconi (dove la Cambiaghi si è formata) alla Cattolica, passando per lo IULM; una carriera "bien remplie", insomma, 
caratterizzata non solo da un'intensa attività didattica, ma anche dalla ricerca che ha costantemente accompagnato tale attività.

La scelta del titolo, che riprende quello del trattato pedagogico di Comenio, Ianua linguarum reserata (1631), non poteva essere più adatta per celebrare chi, come la Cambiaghi, ha dedicato decenni alla linguistica e alla metodologia dell'insegnamento delle lingue (soprattutto francese), alla stregua del grande pedagogo moravo.

Il volume, dopo una rapida presentazione bio-bibliografica della festeggiata, si articola in tre parti: la prima (Le parole e le cose) raccoglie i contributi dedicati alla linguistica generale e alla linguistica francese; la seconda (La didattica), la più ampia e varia delle tre, presenta invece riflessioni di marca schiettamente glottodidattica, spaziando da contributi di natura storica ed epistemologica ad approcci riguardanti metodologie e glottotecnologie; la terza, come indica chiaramente il titolo (Litaliano L2), si concentra sugli aspetti pragmatici della didattica della lingua italiana.

Occorre innanzitutto rilevare la praticità del volume: i saggi sono generalmente brevi, e alcuni non oltrepassano addirittura le 3-4 pagine di lunghezza. La facilità della lettura è inoltre permessa dalla natura descrittiva della maggior parte dei testi. Insomma, traspare una certa volontà pedagogica, e non è certo un caso che la pubblicazione sia avvenuta nell'ambito di una collana destinata a studenti universitari.

Render conto della totalità dei saggi nello spazio angusto di una recensione non è possibile. Mi limito dunque ad accennare a qualche contributo. Il saggio di A. Abi Aad, L'emploi de l'imparfait-mode en italien et en français, analyse énonciative et cognitive, che apre la prima sezione e lo stesso volume, propone una spiegazione interessante per la diffusione dell'imperfetto modale nella protasi ipotetica del periodo della possibilità in francese (si+imp.), adducendo ragioni morfologiche e riflessi cognitivi di base che tendono a stabilire relazioni tra categorie simili. Abi Aad azzarda la stessa spiegazione (con le dovute distinzioni) anche per la diffusione dell'imperfetto, nella medesima situazione sintattica, in italiano (se+imp.). Insomma, il fenomeno sarebbe dovuto a meccanismi di economia linguistica, anche se, l'autore ammette, in italiano l'imperfetto non godrebbe della stessa centralità di cui gode nella lingua francese. Sul finale emerge anche l'aspetto pedagogico di questo castello teorico: la prescrizione delle norme voluta dai grammatici tradizionalisti andrebbe contro il naturale apprendimento delle lingue (del francese e dell'italiano), creando un contrasto tra regole ormai ignorate e la normale evoluzione della lingua. L'autore, tuttavia, sembra astrarre il proprio discorso da una visione diacronica del fenomeno, ignorando che, per l'italiano, tale uso era ben attestato anche in dialoghi teatrali del Cinque, Sei e Settecento (come si afferma in P. Trifone, L'italiano a teatro. Pisa-Roma: IEPI, 2000). Ciò conferma una preesistente tendenza al "modo pragmatico del discorso", parallelo alla norma, e smentisce la visione di un blocco normativo di recente sgretolatosi. Credo che un'indagine in testi antichi francesi riserverebbe sorprese non dissimili.

Nella stessa parte si trova il saggio di M. Rossi e di L. Salmon, Le cas du verlan: stratégies et outils pour la traduction: in modo chiaro e dettagliato è offerta una prospettiva di studio scientifico delle possibilità traduttive del verlan, indicando come le equivalenze lessicali non possano prescindere da precisi contesti culturali. Prendendo spunto dalla resa in italiano della sceneggiatura del premiato (a Cannes) film Entre 
les murs, le due autrici costruiscono con strumenti bibliografici e metodologici un discorso esemplare sulla natura del verlan e della sua forza comunicativa.

Continuando questa veloce carrellata, sono davvero apprezzabili, in mezzo a tanta linguistica pragmatica, i saggi di valore storico presenti nella parte seconda: E. Borello, Glottodidattica e traduzione in Francia tra il 1500 e il 1700; D. Coste, Sur quelques évolutions du dispositif institutionnel d'enseignement du français hors de France de la fin du XIXème à 1940; P. Desideri, Alla scoperta della norma linguistica: "La grammatica di Giannettino» di Carlo Collodi [...].

Nella parte terza, una nota stonata sembra essere il contributo di K. Katerinov, il quale, dietro il titolo Didattica dell'italiano L2 e multidisciplinarità, in realtà tesse la propria 'storia' di docente e di studioso di metodi didattici. Il testo di Katerinov è certo interessante ma, malgrado alcune trovate e spunti, appare sfilacciato e non veramente chiaro nei suoi propositi. Verrebbe da chiedersi se sia davvero necessario scomodare, per quasi due pagine, Einstein e la legge sulla equivalenza di massa-energia solo per affermare la propria indipendenza rispetto a metodologie tradizionali; oppure, quale rapporto ci possa davvero essere tra la didattica e la distanza tra il testo del Placito capuano e la sua fonte orale, o tra la musica suonata e lo spartito.

A fine di volume si segnala il bel saggio di M. Pichiassi, Didattica dell'italiano L2 $e$ in italiano e in italiano L2 ad alunni immigrati: un contesto didattico dalle molte sfide, dato che si tratta di lavorare con non italofoni scolasticamente equiparati agli italofoni.

Per concludere, a livello editoriale, si potrebbe lamentare l'assenza di un indice dei nomi, ma soprattutto di un breve riassunto all'inizio di ogni saggio. Se l'indice appare come un'impresa quasi impossibile, e dai risultati ingombranti, non si capisce invece perché solo qualche contributo sia preceduto da un efficace 'abstract' (con modalità editoriali diverse, peraltro: in corsivo, come primo paragrafo, come veloce premessa che precede il testo, ecc...).

A ogni modo, non si può negare che, grazie a questo volume, la "porta delle lingue" è stata certo aperta, quasi spalancata, invitando altri ad attraversarla.

FRANCO PIERNO

University of Toronto

Pierangela Diadori. Teoria e tecnica della traduzione. Strategie, testi e contesti. Milano: Mondadori Education. 2012. Pp. 380. ISBN 9788800743983. € 28.

Nell'epoca multimediale che mette spesso in seconda fila la lingua, le professioni che si avvalgono del linguaggio verbale hanno subito drammatici mutamenti nell'arco degli ultimi vent'anni. La traduzione è forse l'attività più esposta a sviluppi presenti in tutti i campi dello scibile e dunque più pressante ne è l'aggiornamento. Il volume Teoria e tecnica della traduzione. Strategie, testi e contesti offre un resoconto solido e aggiornato delle conoscenze acquisite per chi si accinge a intraprendere uno studio approfondito di questa disciplina.

Il manuale è diviso in tre parti, precedute dalla Prefazione di Christiane Nord 